\title{
Long-term safety of inhaled corticosteroids in children
}

\author{
S Pedersen \\ From Canadian Society of Allergy and Clinical Immunology Annual Scientific Meeting 2009 \\ Halifax, Canada. 22-25 October 2009
}

Low doses of inhaled corticosteroids control more asthma outcomes to a greater extent than any other asthma therapy in the vast majority of patients and all international guidelines now recommend inhaled corticosteroids as first line treatment in all children who require regular controller therapy for asthma. This paradigm shift in asthma management is based on solid evidence from several controlled trials. Whenever these guideline recommendations are implemented and followed health-care statistics improve markedly in the society. However, several studies have found that often the guideline recommendations are not being followed. There are several reasons for this, including concerns about the safety of long-term use of these agents, particularly in children. These concerns mainly stem from the findings in short-term studies assessing the effects of inhaled corticosteroids on lower leg growth rate or the hypothalamic-pituitary-adrenal axis or markers of bone formation and degradation. However, the clinical relevance of findings in such studies for long-term treatment is unknown and uncertainty exists regarding the predictive value of changes in cortisol levels and clinically relevant changes in long-term growth or bone density.

A recent review on the long-term safety of ICS found that there was some evidence of a small decrease in statural growth rate during the beginning of inhaled corticosteroid therapy. This effect was more marked at daily doses above $200 \mu \mathrm{g}$ and did not apply to all treatment regimens. Studies examining final attained adult height found no difference between patients treated with inhaled corticosteroids and those receives nonsteroidal therapy. None of the studies investigating effects on bone mineral density found any adverse effects of inhaled corticosteroid therapy. Finally, recommended doses of inhaled corticosteroids generally had little or no effect on plasma or urine cortisol levels versus non-steroidal therapy. In contrast, use of short courses of oral steroids (which is common practice in many countries) has been found to increase the risk of fracture in children. The review concluded that recommended doses of inhaled corticosteroids can be administered to children for the long-term management of asthma with minimal risk of clinically relevant adverse effects. This conclusion is supported findings in a study on peak bone mineral density and risk of fracture in 275 ICS treated patients and 161 healthy siblings. The asthma patients had been treated with ICS for 2-22 years (mean 13.7 years) at a mean daily dose of $350 \mu \mathrm{g}$ ICS. In adulthood there was no statistically significant difference between BMD Z-score in ICS treated children (mean Z-score 0.29) and the $\mathrm{z}$ score of their healthy siblings (mean Z-score $=0.34$ ). Moreover, in the asthma group no statistically significant difference was seen between BMD Z -score at study entry $(-0.10)$ and in adulthood (+0.04).

Published: 12 May 2010

doi:10.1186/1710-1492-6-S1-P7

Cite this article as: Pedersen: Long-term safety of inhaled

corticosteroids in children. Allergy, Asthma \& Clinical Immunology 20106 (Suppl 1):P7.

Pediatric Research Unit, Kolding Hospital, DK6000, Kolding, Denmark 